\title{
Minimal radiative Dirac neutrino mass models
}

\author{
Julian Calle, ${ }^{1, *}$ Diego Restrepo, ${ }^{1, \dagger}$ Carlos E. Yaguna, ${ }^{2, *}$ and Óscar Zapata ${ }^{1, \S}$ \\ ${ }^{1}$ Instituto de Física, Universidad de Antioquia, Calle 70 \# 52-21, \\ Apartado Aéreo 1226, Medellín, Colombia \\ ${ }^{2}$ Escuela de Física, Universidad Pedagógica y Tecnológica de Colombia, \\ Avenida Central del Norte \# 39-115, Tunja, Colombia
}

\section{(Received 21 December 2018; published 9 April 2019)}

\begin{abstract}
Neutrinos may be Dirac particles of which the masses arise radiatively at one loop, naturally explaining their small values. In this work, we show that all the one-loop realizations of the dimension-5 operator to effectively generate Dirac neutrino masses can be implemented by using a single local symmetry: $U(1)_{B-L}$. Since this symmetry is anomalous, new chiral fermions, charged under $B-L$, are required. The minimal model consistent with neutrino data includes three chiral fermions, two of them with the same lepton number. The next minimal models contain five chiral fermions, and their $B-L$ charges can be fixed by requiring a dark matter candidate in the spectrum. We list the full particle content as well as the relevant Lagrangian terms for each of these models. They are new and simple models that can simultaneously accommodate Dirac neutrino masses (at one loop) and dark matter without invoking any discrete symmetries.
\end{abstract}

DOI: 10.1103/PhysRevD.99.075008

\section{INTRODUCTION}

The interpretation of neutrino experimental data in terms of neutrino oscillations is compatible with both Majorana and Dirac neutrino masses [1]. The former possibility has received the most attention, but given the lack of signals in neutrinoless double beta decay experiments [2-7], the latter cannot be dismissed. If neutrinos are Dirac particles, the Standard Model (SM) particle content must be extended with right-handed neutrinos, and some symmetry must be imposed to prevent their Majorana mass terms. At least a $Z_{3}$ symmetry is required to guarantee the neutrino Dirac-ness through

$$
\mathcal{L}_{\nu}=y_{D}\left(\nu_{R}\right)^{\dagger} L \cdot H+\text { H.c. },
$$

with ${ }^{1} L \cdot H=\epsilon_{a b} L^{a} H^{b}$, where $L$ is the lepton doublet, $H$ is the SM Higgs doublet with hypercharge $Y=1$, and $y_{D}$ is the matrix of neutrino Yukawa couplings. To be compatible

*julian.callem@udea.edu.co

restrepo@udea.edu.co

\$carlos.yaguna@uptc.edu.co

\$oalberto.zapata@udea.edu.co

${ }^{1}$ Throughout the text, we will follow the convention of defining only left-handed Weyl spinors [8], and we will use the $S U(2)$ metric to build scalar products.

Published by the American Physical Society under the terms of the Creative Commons Attribution 4.0 International license. Further distribution of this work must maintain attribution to the author(s) and the published article's title, journal citation, and DOI. Funded by SCOAP ${ }^{3}$. with neutrino oscillation data [9], $y_{D}$ should be at least of order $2 \times 3$. A possible assignment for the set of SM fields that transform nontrivially under $Z_{3}$ is $L \sim \omega,\left(e_{R}\right)^{\dagger} \sim \omega^{2}$, and $\left(\nu_{R}\right)^{\dagger} \sim \omega^{2}$, with $\omega^{3}=1$. At this level, the neutrino mass problem is not longer a phenomenological issue but a theoretical one, in which it is necessary to explain the smallness of the Yukawa couplings in $y_{D}$, which must be of order $10^{-11}$.

To do so, we assume that the symmetry allows for the five-dimensional operator with total lepton number conservation [10]

$$
\mathcal{L}_{5}=\frac{h}{\Lambda}\left(\nu_{R}\right)^{\dagger} L \cdot H S^{*}+\text { H.c. },
$$

where $\Lambda$ is the new physics scale, and that this operator is first realized at one-loop level [11] (see Refs. [11-19] for the tree-level realizations).

Regarding the symmetry, we follow the usual approach of promoting baryon number $(B)$ minus lepton number $(L)$ from an accidental global symmetry of the SM, to a local Abelian symmetry, $U(1)_{B-L}$, which is spontaneously broken. One of the main novelties of our work is that we do not impose any other symmetries, discrete or otherwise. Thus, the charges of the right-handed neutrinos under $U(1)_{B-L}$ should be such that the tree-level Dirac mass term (1) is forbidden. This requirement automatically excludes the usual assignment where the three right-handed neutrinos have $B-L$ charges equal to -1 .

The classification of all the topologies at one-loop level that realize the effective operator (2) has been presented in 
Ref. [11]. There, in addition to the $U(1)_{B-L}$ symmetry, at least one additional $Z_{2}$ symmetry was imposed to avoid the Majorana mass terms for the right-handed neutrinos, and a further $Z_{2}^{\prime}$ was required to avoid $i$ ) the appearance of treelevel realizations in the cases when $U(1)_{B-L}$ is not able to do it and ii) to have a dark matter candidate in the particle spectrum-one of the new particles needed to realize (2). Here, we focus instead on the simplest realizations of each topology that can be realized with a single symmetry, $U(1)_{B-L}$. This same symmetry would be responsible for the stability of possible dark matter candidates appearing in the different realizations. Let us stress that until now in the literature there has not been a simple realization of operator (2) at one loop invoking only a single symmetry. There have been some efforts in this direction, but either some Majorana terms were left out, which would need to be forbidden with an extra $Z_{2}$-symmetry [20,21], or the found models require many extra fields [20].

Using only $U(1)_{B-L}$, we find, for each topology, the realization with the minimum number of fields. The minimal model requires three chiral fields, two of them sharing the same lepton number, so that the spectrum contains two massive Dirac neutrinos. The next minimal models include five chiral fields. Interestingly, their $B-L$ charges are fixed once the requirement to have a dark matter particle is imposed. Hence, these new models may account for dark matter and Dirac neutrino masses (at one loop) without invoking any discrete symmetries.

The rest of the paper is organized as follows. In the next section, we introduce the notation and derive the conditions necessary to realize the different topologies that give rise to one-loop Dirac neutrino masses. Our main results are presented in Sec. III. There, the particle content of the minimal models, with three and five chiral fields, is spelled out. Finally, in Sec. IV, our conclusions are drawn.

\section{GENERAL SETUP}

We use the notation for the topologies defined in Ref. [11], which are displayed in Fig. 1. There the flux of the lepton number is illustrated by the wide colored arrows. The green arrow represents the flux of the doublet lepton number, $L\left(L_{i}\right)=-1$; the yellow is for $L\left(\nu_{R \beta}\right)=-\nu$ with $\beta$ at least 1,2 ; the blue is for $L(S)=s$; and the red is for some internal circulating $L$ charge associated with a chiral fermion, which we choose as a free parameter in our setup.

We do not consider the T1-1 or T4 topologies of Ref. [11] because the former is already included in topology T3-1 when the $X_{3}$ scalar field is decoupled, whereas the latter requires further symmetries to forbid the tree-level contribution. ${ }^{2}$ In Ref. [11], it was assumed that the internal

\footnotetext{
${ }^{2}$ The exception cases are the IV and V solutions of T4-3-I. However, they require mixing between the charged leptons and the new fermion fields, thus leading to charged lepton flavor violation processes which are quite constrained.
}

fermion lines were already vectorlike fermions, which allow them to use the $\nu=1$ solution for anomaly cancellation conditions, but they needed to impose additional $Z_{2}$ symmetries to forbid the tree-level contribution to neutrino masses and to stabilize the dark matter. Here, we instead assume chiral fields for the fermions that are singlets under the SM gauge group. This allows us to search for new minimal realizations of the topologies with a single extra symmetry beyond the SM. It is clear that our solution with three chiral states, corresponding to the three right-handed neutrinos, can be easily extrapolated to all the solutions found in Ref. [11], with the advantage that not further discrete symmetries are imposed. We explicitly illustrate this for the case of topology T3-1-A, the minimal solution of which involves just a Dirac fermion.

In our setup, the only extra symmetry beyond the SM, $U(1)_{B-L}$, must forbid the tree-level terms

$$
\mathcal{L}_{\nu}=y_{\beta i}^{D}\left(\nu_{R \beta}\right)^{\dagger} L_{i} \cdot H+M_{\beta \gamma}^{R}\left(\nu_{R \beta}\right)^{\dagger}\left(\nu_{R \gamma}\right)^{\dagger} S+\text { H.c }
$$

as well as allow for the dimension-5 operator (2). This is accomplished by the $L$ charge assignments in Table I. There, $\nu$ is the lepton number of the left-handed antineutrino, which is common to at least the two right-handed neutrinos required to explain the neutrino oscillation data. As already mentioned, the solution with $\nu=1$, studied in Ref. [11], is no longer considered in this work.

To find the new possible solutions, we explore the anomaly cancellation conditions with five chiral fields by checking that their charges do not generate direct or induced Majorana mass terms for them; that is, the fermion loop mediators are Dirac-like fields. In addition to the chiral fields for $\beta=1,2$, we introduce $\left(\nu_{R k}\right)^{\dagger}$ with $L\left(\nu_{R k}\right)=-\nu_{k}$ and a heavy Dirac fermion field with Weyl components $\psi_{L}$ and $\left(\psi_{R}\right)^{\dagger}$, such that $L\left(\psi_{L}\right)=l$ and $L\left(\psi_{R}\right)=-r$, respectively. The linear and cubic anomaly cancellation conditions are $[22,23]$

$$
2 \nu+\nu_{k}+l+r=3, \quad 2 \nu^{3}+\nu_{k}^{3}+r^{3}+l^{3}=3 .
$$

From the linear equation,

$$
\nu_{k}=3-2 \nu-l-r .
$$

To further proceed, we separate the topologies in two types: the set (A) with T1-3-D and T3-1-A, corresponding to the ones with the yellow $\nu$ flux in Fig. 1 and where $S$ is in a vertex only involving scalars, and the set (B) with T1-3-E and T1-2-(A/B), with the blue $s$ flux in Fig. 1 and where $S$ is in a Yukawa-type vertex. For each case, we have

(A) The heavy Dirac fermion has a vectorlike mass, such that

$$
l=-r .
$$




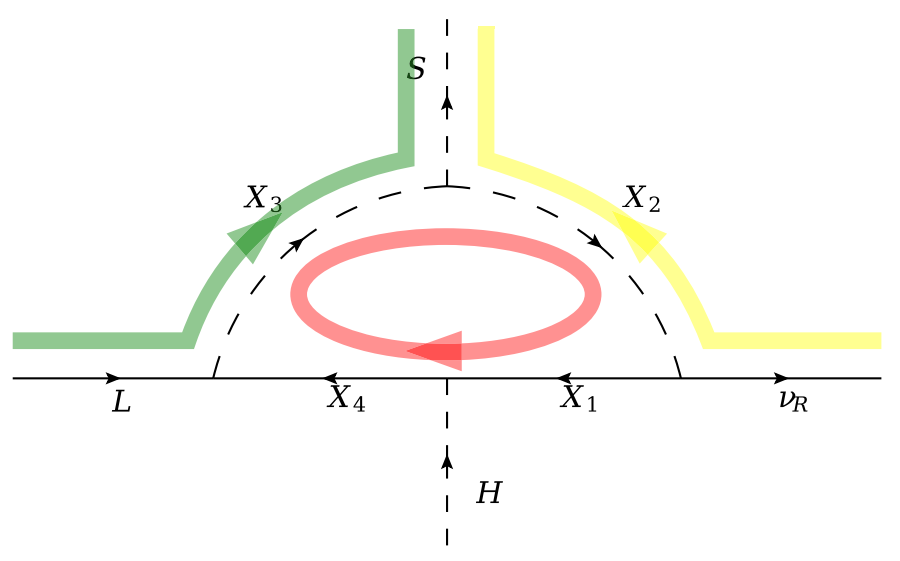

T1-3-D

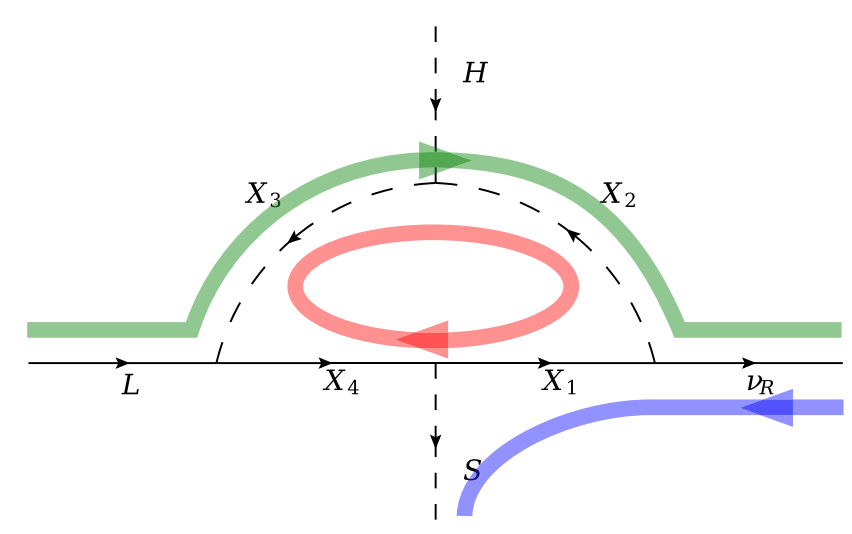

T1-3-E

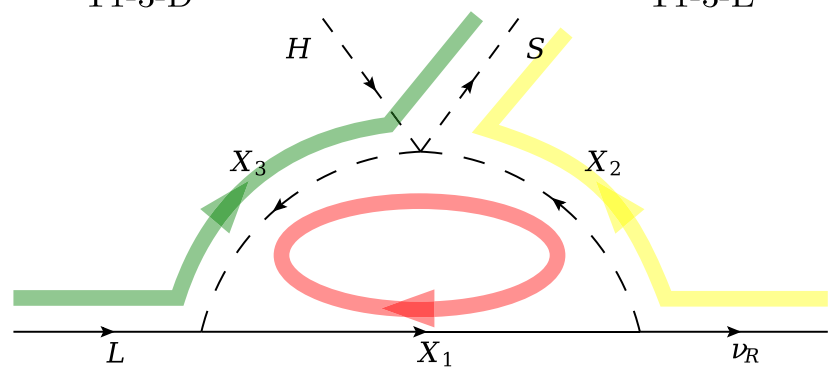

T3-1-A

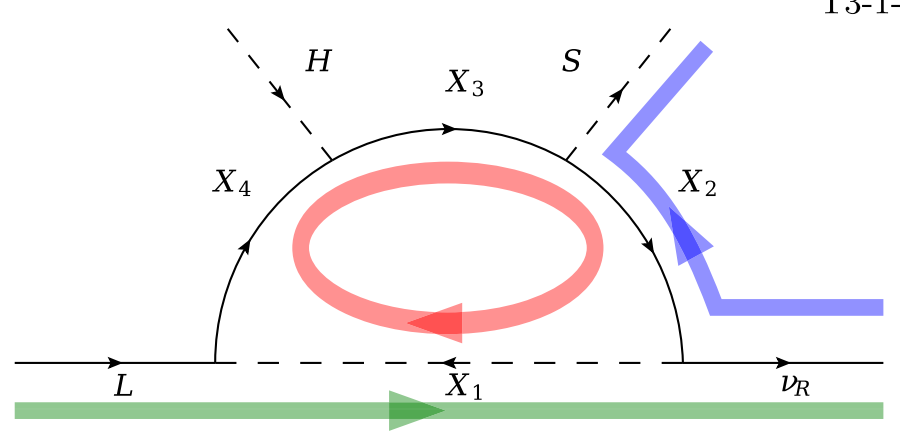

T1-2-A

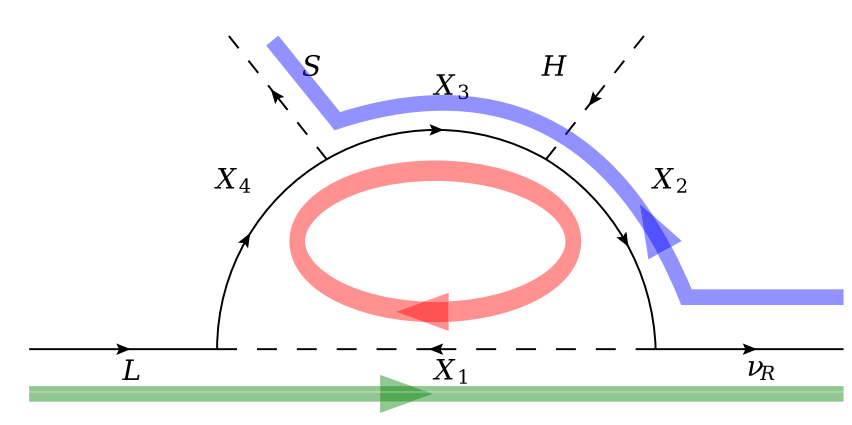

T1-2-B

FIG. 1. Topologies (in notation of Ref. [11]) leading to one-loop Dirac neutrino masses.

Replacing back in Eq. (5), we obtain

$$
\nu_{k}=3-2 \nu
$$

Solving the cubic equation for $\nu$ gives rise to two different roots: 1 and 4. Thus, we choose $\nu=4$, which leads to $\nu_{k}=-5$.

On the other hand, the fact that the new fermion field $\psi$ does not contribute to the anomalies implies that the only possible realization within the T1-3-D topology is the solutions I and II with $\psi_{L}=L_{i}$ and $\psi_{R}=e_{R i}$, since in these cases the corresponding contributions to the anomalies are already taken into account.

(B) The two SM-singlet chiral fields can acquire a Dirac mass [after the spontaneous symmetry breaking (SSB) of $U(1)_{B-L}$ ] through

$$
\mathcal{L}_{\psi}=h_{S}\left(\psi_{R}\right)^{\dagger} \psi_{L} S+\text { H.c.. }
$$

If we choose $r$ as the free charge circulating in the loop, and since from Table I we have $s=\nu-1$, then from the condition in Eq. (8), $r+l+s=0$, we get

$$
l=1-\nu-r
$$

and replacing back in Eq. (5)

TABLE I. General assignment of lepton number for external legs of the one-loop topologies in Fig. 1.

\begin{tabular}{lcccc}
\hline \hline Fields & $H$ & $L_{i}$ & $\left(\nu_{R \beta}\right)^{\dagger}$ & $S$ \\
\hline$L$ & 0 & -1 & $\nu \neq 1$ & $\nu-1 \neq-2 \nu$ \\
\hline \hline
\end{tabular}




$$
\nu_{k}=2-\nu .
$$

Using (9) and (10) in the cubic condition for anomaly cancellation, Eq. (4), we end up with the one-parameter solution,

$$
\nu=\frac{r^{2}-r+2}{3-r} .
$$

If $\nu_{k}=0$, we would have a solution with four chiral fields when $\nu=2$; however, the required $r$ charge is irrational and will not be further considered here.

To label the solutions, we use the conventions of Ref. [11], as Ta-n-b-I $\alpha$, where a refers to the topology itself, $\mathrm{n}$ indicates the different choices of the fermion and scalar lines in a given topology, b denotes the field assignments for external fields, I denotes the simplest solution with standard model-singlet scalars or fermions, and $\alpha$ is the parameter which fixes the hypercharges of the $X_{i}$ fields inside the loop.

It is worth mentioning that when both $\psi_{L}$ and $\psi_{R}$ are SM singlets (in the T3-1-A-I with $\alpha=0$, T1-3-E-I with $\alpha=0$, T1-2-A-I with $\alpha=0$, and T1-2-B-II with $\alpha=+1$ models), the condition $r \neq 1$ or $l \neq-1$ must be imposed in order to avoid the tree-level realization of (2) that involves a SMsinglet fermion mediator-the so-called type I Dirac seesaw. In this way, one of the two required terms in that realization $\left(\psi_{R}^{\dagger} L_{i} \cdot H\right.$ and $\left.\nu_{R \beta}^{\dagger} \psi_{L} S^{*}\right)$ is forbidden.

As usual for scotogenic models, we demand the lightest neutral particle running in the loop to be stable. For the case of scalar dark matter (DM), the stability is guaranteed if there is no linear terms in the scalar potential involving scalar loop mediators, neither Yukawa interactions with two SM fermions. The minimal DM scenarios that may arise are then the singlet [24-26], doublet [27,28], and singlet-doublet [29-32] scalar DM. It is worth mentioning that, since Majorana mass terms for the fermion loop mediators are not allowed, the fermion DM candidate is either singlet [33] or singlet-doublet Dirac DM [34].

The wanted solutions must satisfy the constraints regarding DM stability and Dirac-ness of light neutrinos and guarantee that the direct or induced fermion mass terms between the right-handed neutrinos and $\psi_{L}$ or $\psi_{R}$ are forbidden. The reason to exclude this kind of mixings is that in such a case the DM would not be stable because the fermion loop mediator would decay into particles of the visible sector. For instance, the induced (through $S$ ) or direct mixing between $\left(\nu_{R k}\right)^{\dagger}$ and either $\psi_{L}$ or $\left(\psi_{R}\right)^{\dagger}$ leads to the decay into $S$ and $\nu_{R k}$ for the induced mixing and into $Z_{\mu}^{\prime}$ and $\nu_{R k}$ for the direct mixing.

The solutions of the two sets are displayed in Table II. Solution (A) is the well-known one studied in Ref. [15] for tree-level realization of the five-dimensional operator. In this solution, $r$ is quite free; in fact, $r= \pm 1 / 2, \pm 1 / 3, \ldots$.
TABLE II. Solutions for Dirac neutrino masses with Dirac loop mediators for $i \neq j \neq k$.

\begin{tabular}{lccccccc}
\hline \hline Fields & & $\left(\nu_{R i}\right)^{\dagger}$ & $\left(\nu_{R j}\right)^{\dagger}$ & $\left(\nu_{R k}\right)^{\dagger}$ & $\psi_{L}$ & $\left(\psi_{R}\right)^{\dagger}$ & $S$ \\
\hline$L$ & (A) & +4 & +4 & -5 & $-r$ & $r$ & +3 \\
& (B) & $+\frac{8}{5}$ & $+\frac{8}{5}$ & $+\frac{2}{5}$ & $\frac{7}{5}$ & $-\frac{10}{5}$ & $+\frac{3}{5}$ \\
\hline \hline
\end{tabular}

The solution (B) was obtained after exploring all the solutions of Eq. (11) for rational values of $|r| \leq 10$, and with both the numerator and denominator less than or equal to 10 . Since the anomaly cancellation conditions are invariant under the exchange of $r$ with $l$, a second solution for (B) exists with the charges of $\psi_{L}$ and $\left(\psi_{R}\right)^{\dagger}$ exchanged.

Higher $S U(2)_{L}$ fermion representations, as required in T1-2 topologies, need to be introduced as vectorlike fermions to not spoil the anomaly cancellation conditions of the standard model. We will denote vectorlike doublet Weyl fermions fields with $Y=-1 \quad(Y=+1)$ as $\Psi_{L, R}\left(\Upsilon_{L, R}\right)$.

Regarding the scalars circulating in the loop, we will use $\sigma$ and $\eta$ to represent $S U(2)_{L}$ scalar singlets and scalar doublets, respectively, and we will denote their nonzero lepton number with the same symbols.

A final comment is in order. Because $U(1)_{B-L}$ is promoted to a gauge symmetry, the vacuum expectation value of $S,\langle S\rangle=v_{S} / \sqrt{2}$, induces a nonzero mass to the associated gauge boson $Z_{B L}$. The expression for its mass can be cast as $M_{Z_{B L}}=g_{B L} v_{S}|s|$, where $g_{B L}$ is the $B-L$ gauge coupling and $s$ is the $B-L$ charge of $S$. On the other hand, since $Z_{B L}$ couples to all the SM fermions (they have nonzero $B-L$ charges), it can be produced in hadron and lepton colliders leading to observable signatures. Indeed, from the nonobservation of any of such signatures in the large electron positron and LHC data, there exist constraints on its mass and gauge coupling [35-39] (see e.g., Refs. [20,21] for specific analysis in $B-L$ scotogenic Dirac models).

\section{SOLUTIONS}

The new solutions correspond to the case in which some of the $X_{i}$ fermion fields in Fig. 1 can be chosen as chiral fields. We explore the solutions with the minimal number of fermion fields beyond the standard model. We are interested, therefore, in the solutions in which at least two right-handed neutrinos have the same $U(1)_{B-L}$ charge because in such a case both of them can couple to the same set of extra chiral fermions. All the solutions for the $B-L$ charges presented below have been chosen in such a way the DM particle does not decay.

\section{A. Chiral T1-3-D-I $(\alpha=-2)$}

We will start our analysis with the case in which an internal fermion line in Fig. 1 can be interpreted as a 


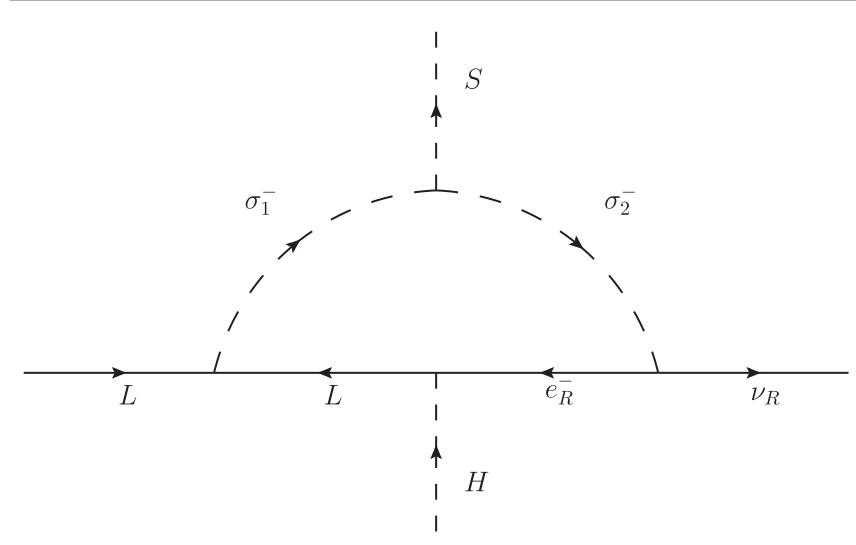

Chiral T1-3-D-I $(\alpha=-2)$

FIG. 2. Dirac Zee model.

standard model field. Therefore, only the right-handed neutrinos contribute to the anomaly cancellation conditions of the SM with $U(1)_{B-L}$. There are three well-known solutions with three chiral fields $[15,40]$. However, solution (A) in Table II is the only one that satisfies our constraints.

In fact, with the additions of two charged scalars, $\sigma_{1,2}^{ \pm}$, which are singlet under $S U(2)_{L}$, we can build the Dirac version of the Zee mechanism to generate neutrino masses [41]. The couplings required to build the diagram displayed in Fig. 2 are

$$
\begin{aligned}
\mathcal{L} \subset & {\left[f_{i j} L_{i} \cdot L_{j} \sigma_{1}^{+}+h_{e}^{i j}\left(e_{R i}\right)^{\dagger} L_{j} \cdot \tilde{H}+h_{R}^{i \beta} e_{R i} \nu_{R \beta} \sigma_{2}^{+}+\text {H.c. }\right] } \\
& +V\left(\sigma_{1}^{ \pm}, \sigma_{2}^{ \pm}, H\right),
\end{aligned}
$$

where $L_{i}$ are the SM lepton doublets, $H=\left(H^{+}, H^{0}\right)^{T}$, $\tilde{H}=i \sigma_{2} H^{*}$, and $V\left(\sigma_{1}^{ \pm}, \sigma_{2}^{ \pm}, H\right)$ is the scalar potential.

Therefore, $\psi_{L}\left(\psi_{R}\right)$ in solution (A) of Table II corresponds to three lepton doblets $L_{i}$ (right-handed electrons $\left.e_{R i}^{-}\right)$with $l=-1(r=1)$ as the usual lepton number. Since we use one set of charged scalar fields, $\sigma_{1,2}^{ \pm}$, the model has two massless chiral fields, one of them, $\nu_{R k}$, contributing to the effective number of relativistic degrees of freedom, $N_{\text {eff }}$ $[21,40,42]$. From the lepton number flux in Fig. 2, we have

$$
L\left(\sigma_{1}^{+}\right)=-2, \quad L\left(\sigma_{2}^{+}\right)=-5 .
$$

The full solution is presented in Table III. This is by far the minimal model for Dirac neutrino masses with a gauged $U(1)_{B-L}$. The Dirac Zee model with $\nu=\nu_{k}=1$ and extra discrete symmetries has been studied in Ref. [41].

It is worth noticing that the restrictions from $N_{\text {eff }}$ are expected to be stronger in our model because of the larger lepton number assignment for the right-handed neutrinos. However, since they do not couple directly to any SM particles, we can simply assume that their interactions with the extra gauge boson and scalars are sufficiently suppressed that they decouple early enough from the thermal
TABLE III. Chiral T1-3-D-I $(\alpha=-2)$ : Solutions for the Dirac Zee model with $i \neq j \neq k(i, j, k, l=1,2,3)$.

\begin{tabular}{lccccccccc}
\hline \hline Fields & & & & $X_{1}$ & $X_{2}$ & $X_{3}$ & $X_{4}$ & \\
T1-3-D-I & & $\left(\nu_{R i}\right)^{\dagger}$ & $\left(\nu_{R j}\right)^{\dagger}$ & $\left(\nu_{R k}\right)^{\dagger}$ & & & & & $S$ \\
\hline$L$ & & & $e_{R_{l}}^{-}$ & $\sigma_{2}^{-}$ & $\sigma_{1}^{-}$ & $L_{l}$ & \\
\hline \hline
\end{tabular}

bath. On the other hand, it is clear that there is not a DM candidate in this model. Indeed, this is just a specific example of models with one-loop Dirac neutrino masses but without a DM candidate that can be obtained within our setup.

Regarding $\nu_{R k}$, it can give rise to either a third Dirac neutrino mass if we extend the scalar sector with $S^{\prime}$ and $\sigma_{2}^{\prime \pm}$ of $L$ charges -6 and +8 , respectively, or a Majorana dark matter candidate if we extend the scalar sector with a $S^{\prime}$ of $L$ charge +10 [40]. In both cases, we end up with a physical Goldstone boson (GB) which could contribute to $N_{\text {eff }}$ through interactions with the Higgs [43]. The conditions that GB decouples from the bath in the early Universe are analyzed in Ref. [40] and require couplings of GB with SM Higgs not larger than $10^{-3}$. This discussion can be easily extended to the other one-loop realizations below.

We have implemented the model with three nonzero Dirac neutrino masses in SARAH [44]. We use the method in Refs. [41,45] to express $f_{13}, f_{23}, h_{11}, h_{22}, h_{33}^{R}, h_{31}^{R}, h_{32}^{R}$, and $h_{23}^{R}$ as a function of the neutrino masses and mixings, by using $f_{12}^{R}, h_{12}^{R}, h_{13}^{R}$, and $h_{21}^{R}$ as free parameters. For an example of the consistency of the model, we show in Fig. 3 the observable $\operatorname{Br}\left(\mu^{+} \rightarrow e^{+} \gamma\right)<4.2 \times 10^{-13}$ [46] as a function a $f_{12}$. The other parameters were fixed as $\theta=0.1, \quad M_{\sigma_{1}}=500 \mathrm{GeV}, \quad M_{\sigma_{2}}=750 \mathrm{GeV}, \quad h_{12}^{R}, \quad h_{13}^{R}$, $h_{21}^{R}=10^{-4}$, where $\theta$ is the mixing angle between the charged mass eigenstates $\sigma_{1}$ and $\sigma_{2}$. We can see that the value for the parameter $f_{12}$ is restricted to values lower than 0.02 .

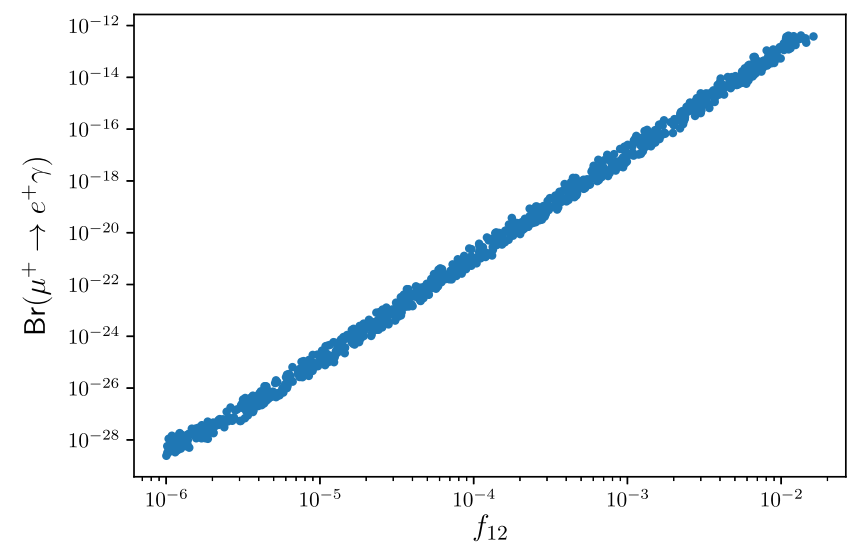

FIG. 3. Relationship between $\operatorname{Br}\left(\mu^{+} \rightarrow e^{+} \gamma\right)$ and the parameter $f_{12}$. 


\section{B. Chiral T1-3-E-I $(\alpha=0)$}

We consider now the topologies where two SM-singlet chiral fields acquire a Dirac mass after the SSB of $U(1)_{B-L}$ from the term $\mathcal{L}_{\psi}$ in Eq. (8). For them, we will use the solution (B). The relevant terms in the Lagrangian include

$$
\mathcal{L} \supset \mathcal{L}_{\psi}+\mathcal{L}_{\sigma \psi}+\mathcal{L}_{\eta \psi}+V\left(\sigma_{a}, \eta_{a}, S, H\right)
$$

where $\mathcal{L}_{\psi}$ was given in Eq. (8),

$$
\begin{aligned}
& \mathcal{L}_{\sigma \psi}=h_{1}^{\beta a}\left(\nu_{R \beta}\right)^{\dagger} \psi_{L} \sigma_{a}^{*}+\text { H.c. }, \\
& \mathcal{L}_{\eta \psi}=h_{1}^{\prime i a}\left(\psi_{R}\right)^{\dagger} L_{i} \cdot \eta_{a}+\text { H.c. }
\end{aligned}
$$

and $V\left(\sigma_{a}, \eta_{a}, S, H\right)$ is the scalar potential.

After the spontaneous breaking of the $U(1)_{B-L}$ symmetry, this topology is reduced to the well-known Dirac radiative seesaw model, but with different Lepton number assignments. In fact, the model with $\nu=\nu_{k}=r=1$ and two extra $Z_{2}$ discrete symmetries was first introduced in Refs. [47,48], while the case with $r \neq 1$ which requires only one extra $Z_{2}$ symmetry was studied in Ref. [49]. The minimal set of fermion fields is achieved when the two nonzero Dirac neutrino masses are generated with two sets of SM-singlet and doublet scalars: $\eta_{a}, \sigma_{a}$ [49].

From Fig. 4, the charges of the scalars are

$$
\eta=1-r, \quad \sigma=1-r .
$$

The solution compatible with the fields in Fig. 4 is displayed in Table IV. The phenomenology of the radiative seesaw model with $\nu=1$ has been already studied in the literature $[20,21,47-49]$, where either singlet Dirac $(\psi)$ or singlet-doublet scalar $\left(\sigma^{a}, \eta^{a}\right)$ DM is realized. Because of the similar charges associated to solution (B), we do not expect significant differences with respect to those works.

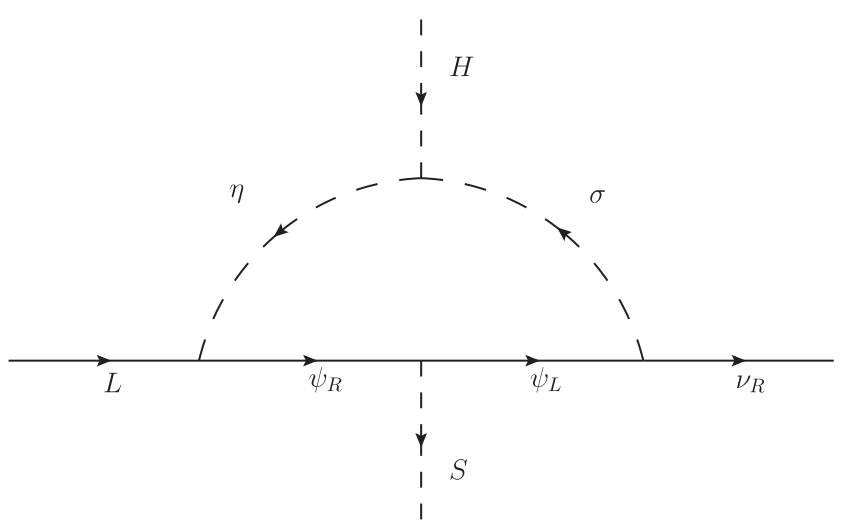

T1-3-E-I $(\alpha=0)$

\begin{tabular}{|c|c|c|c|c|c|c|c|c|c|}
\hline Fields & $\left(\nu_{R i}\right)^{\dagger}$ & $\left(\nu_{R j}\right)^{\dagger}$ & $\left(\nu_{R k}\right)^{\dagger}$ & $X_{1}$ & & & & $X_{4}$ & \\
\hline T1-3-E-I & & & & $\psi_{L}$ & $\eta_{a}$ & & $\sigma_{a}$ & $\psi_{R}$ & \\
\hline$L$ & & & & $\frac{7}{5}$ & $\frac{15}{5}$ & & $\frac{15}{5}$ & $\frac{10}{5}$ & \\
\hline T1-2-A-I & & & & $\sigma_{a}$ & $\psi_{R}$ & & $\psi_{L}$ & $\left(\widetilde{\left.\Psi_{R}\right)}\right.$ & $\Psi_{L}$ \\
\hline$L$ & $\frac{8}{5}$ & $\frac{8}{5}$ & $\frac{2}{5}$ & $\frac{15}{5}$ & $\frac{10}{5}$ & & $\frac{7}{5}$ & $-\frac{10}{5}$ & $\frac{10}{5} \quad \frac{3}{5}$ \\
\hline T1-2-B-II & & & & $\eta_{a}$ & $\left(\widetilde{\Upsilon_{R}}\right)$ & & $\psi_{R}$ & $\psi_{L}$ & \\
\hline$L$ & & & & $\frac{15}{5}$ & $-\frac{7}{5}$ & $\frac{7}{5}$ & $\frac{10}{5}$ & $\frac{7}{5}$ & \\
\hline
\end{tabular}

FIG. 4. T1-3-E-I $(\alpha=0)$.
TABLE IV. Chiral solutions for Dirac neutrino masses with the minimal $\mathrm{T} 1$ topologies with $i \neq j \neq k$ and $\alpha=0$.

\section{Chiral T1-2-A-I $\alpha=0$}

The solution compatible with the fields in Fig. 5 (left), requires at least the following terms in the Lagrangian,

$$
\begin{aligned}
\mathcal{L} \supset & \mathcal{L}_{\psi}+\mathcal{L}_{\sigma \psi}+\left[M _ { \Psi } \left(\widetilde{\left.\Psi_{R}\right)} \cdot \Psi_{L}+h_{2}^{i a}\left(\widetilde{\left.\Psi_{R}\right)} \cdot L_{i} \sigma_{a}\right.\right.\right. \\
& \left.+y_{1}\left(\psi_{R}\right)^{\dagger} \Psi_{L} \cdot H+\text { H.c. }\right]+V\left(\sigma_{a}, S, H\right),
\end{aligned}
$$

where $\Psi_{L}=\left(\Psi_{L}^{0}, \Psi_{L}^{-}\right)^{\mathrm{T}},\left(\widetilde{\left.\Psi_{R}\right)}=\left(\left(\Psi_{R}^{-}\right)^{\dagger},-\left(\Psi_{R}^{0}\right)^{\dagger}\right)^{\mathrm{T}}\right.$, and $V\left(\sigma_{a}, S, H\right)$ is the scalar potential. It follows that this model requires a set of at least two SM-singlet scalars to generate a rank-2 neutrino mass matrix and allows for either singlet scalar $\left(\sigma^{a}\right)$ or singlet-doublet Dirac $(\psi, \Psi)$ DM.

From Fig. 5 (left),

$$
\sigma=1-r
$$

The corresponding charges for the solution (B) are shown in Table IV.

\section{Chiral T1-2-B-II $\alpha=0$}

The solution compatible with the fields in Fig. 5 (right) requires at least the following terms in the Lagrangian,

$$
\begin{aligned}
\mathcal{L} \supset & \mathcal{L}_{\psi}+\mathcal{L}_{\eta \psi}+\left[M _ { \Upsilon } \left(\widetilde{\left.\Upsilon_{R}\right)} \cdot \Upsilon_{L}+h_{2}^{\prime a \beta}\left(\widetilde{\left.\Upsilon_{L}\right)} \cdot \eta_{a} \nu_{R \beta}\right.\right.\right. \\
& \left.+y^{\prime}{ }_{1} \widetilde{\Upsilon_{R}} \cdot H \psi_{L}+\text { H.c. }\right]+V\left(\eta_{a}, S, H\right),
\end{aligned}
$$

where $\Upsilon_{L}=\left(\Upsilon_{L}^{+}, \Upsilon_{L}^{0}\right)^{\mathrm{T}},\left(\widetilde{\left.\Upsilon_{R}\right)}=\left(\left(\Upsilon_{R}^{0}\right)^{\dagger},-\left(\Upsilon_{R}^{+}\right)^{\dagger}\right)^{\mathrm{T}}\right.$, and $V\left(\eta_{a}, S, H\right)$ is the scalar potential. It follows that this model requires a set of at least two scalar doublets to generate a rank-2 neutrino mass matrix and allows for either doublet scalar $\left(\eta^{a}\right)$ or singlet-doublet Dirac $(\Upsilon, \psi)$ DM.

From the figure,

$$
\eta=1-r
$$

The solutions compatible with the fields in Fig. 5 (right) correspond to the ones displayed in Table IV.

The phenomenological analysis of the chiral realizations of topologies T1-2 will be done elsewhere. It is worth 


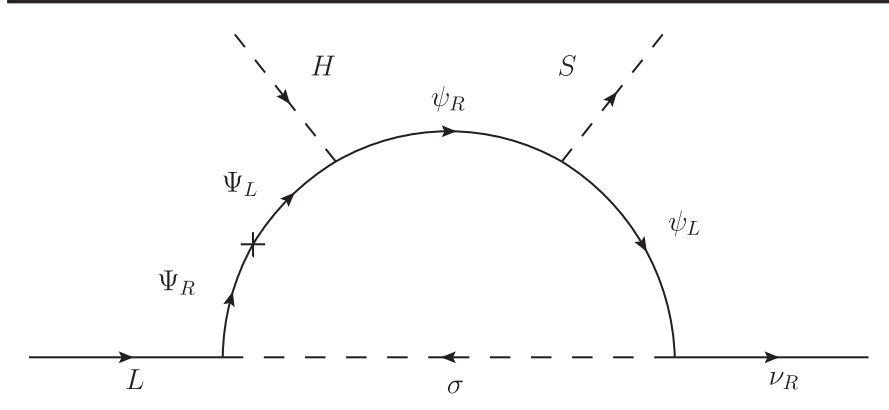

Chiral T1-2-A-I $(\alpha=0)$

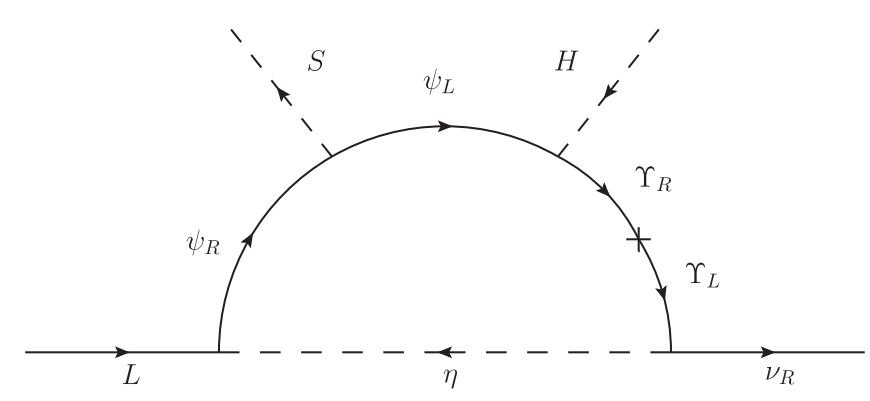

Chiral T1-2-B-II $(\alpha=0)$

FIG. 5. Chiral T1-2-A-I $(\alpha=0)$ Chiral T1-2-B-II $(\alpha=0)$.

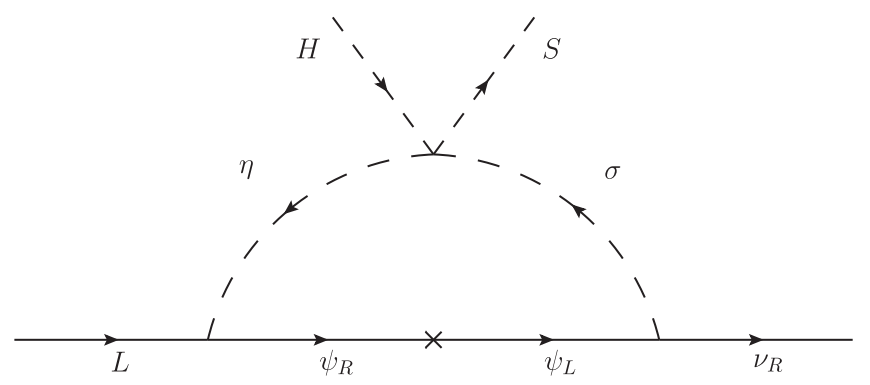

FIG. 6. T3-1-A-I $(\alpha=0): B-L$ flux in the Dirac radiative seesaw.

noting, however, that a model independent analysis of the effect of the right-handed neutrinos on $N_{\text {eff }}$ can be inferred from Ref. [42]. The ratio $M_{Z_{B L}} /\left(\nu g_{B L}\right)$, where $g_{B L}$ is the $U(1)_{B-L}$ gauge coupling, must be larger than $7-8 \mathrm{TeV}$ in order to be in agreement with the cosmological constraints.

\section{E. Vectorlike solutions}

In Ref. [11], all the obtained solutions for the topologies in Fig. 1 have internal vectorlike fermions. We want to stress that it is possible to realize all of them with a single extra symmetry $U(1)_{B-L}$. For that, we can use the solution (A) with a proper choice of the circulating free charge $r$.

For example, in the simplest case of just one circulating SM-singlet Dirac fermion line as in T3-1-A, we have the diagram displayed in Fig. 6.

From the flux of lepton number in Fig. 6, we have

$$
\eta=1-r, \quad \sigma=\nu-r .
$$

TABLE V. T3-1-A-I $(\alpha=0)$ : Solutions for Dirac radiative seesaw model with $i \neq j \neq k(i, j, k=1,2,3)$.

\begin{tabular}{lcccccccc}
\hline \hline Fields & & & & $X_{1}$ & & $X_{2}$ & $X_{3}$ & $X_{4}$ \\
& $\left(\nu_{R i}\right)^{\dagger}$ & $\left(\nu_{R j}\right)^{\dagger}$ & $\left(\nu_{R k}\right)^{\dagger}$ & & & & & $S$ \\
T3-1-A-I & & & $\left(\psi_{R}\right)^{\dagger}$ & $\psi_{L}$ & $\eta_{a}$ & $\sigma_{a}$ & - \\
\hline$L$ & +4 & +4 & -5 & $r$ & $-r$ & $1-r$ & $4-r$ & -3 \\
\hline \hline
\end{tabular}

The corresponding charges for solution (A) are shown in Table V.

Since the charges of the right-handed neutrinos are now bigger than those in solution (B) used in Sec. III B, the constraints from $N_{\text {eff }}$ are expected to be stronger. In fact, a recent detailed phenomenological analysis of the Dirac radiative seesaw [21] includes the case of a right-handed neutrino with $\nu=4$ and can be fully applied here. In particular, the restrictions from $N_{\text {eff }}$ for both scalar $\left(\sigma^{a}, \eta^{a}\right)$ and Dirac fermion $(\psi)$ dark matter cases are more important than the restriction from $Z_{B L}$ searches at the LHC. We refer the reader there for further details.

It is clear that after SSB of $U(1)_{B-L}$ both solutions T1-3E-I $(\alpha=0)$ in Sec. III B and T3-1-A-I $(\alpha=0)$ reduce to the Dirac radiative seesaw. Moreover, we can add extra circulating charges in the loop. In fact, when all the circulating particles in the loop are color octets [49], we can have a bound state dark matter candidate formed by two Dirac color-octet fermions [50]. With our solutions, the extra $Z_{2}$ symmetry in Ref. [49] is no longer required.

\section{CONCLUSIONS}

We found new and simple models for Dirac neutrino masses within an extension of the Standard Model by a spontaneously broken $U(1)_{B-L}$ gauge symmetry. Specifically, we studied the minimal chiral realizations, at one loop, of the dimension-5 total lepton number conserving operator that gives rise to Dirac neutrino masses without imposing extra symmetries. The minimal models contain three or five chiral fields, two of them with the same charges under $B-L$. In the latter case, their charges can be fixed by the requirement to have a dark matter particle in the spectrum. The full particle content as well as the relevant Lagrangian terms were given for each of these models. We also showed that known solutions with vectorlike fermions can be obtained with just the single symmetry $U(1)_{B-L}$. These new models, therefore, can simultaneously accommodate one-loop Dirac neutrino masses and dark matter without invoking any discrete symmetries. 


\section{ACKNOWLEDGMENTS}

Work was supported by Sostenibilidad-UdeA and by COLCIENCIAS through Grants No. 111565842691 and No. X111577657253. D. R. thanks Martin Hirsch for illuminating discussions. O.Z. acknowledges the ICTP Simons associates program and the kind hospitality of the Abdus Salam ICTP where part of this work was done.

Note added.-Recently, a related study [51] appeared. The authors also present the solution in Sec. III E (Table V) by fixing $r=1 / 2$.
[1] M. Tanabashi et al. (Particle Data Group), Review of Particle Physics, Phys. Rev. D 98, 030001 (2018).

[2] A. Gando et al. (KamLAND-Zen Collaboration), Search for Majorana Neutrinos Near the Inverted Mass Hierarchy Region with KamLAND-Zen, Phys. Rev. Lett. 117, 082503 (2016); Erratum, Phys. Rev. Lett. 117, 109903(E) (2016).

[3] M. Agostini et al. (GERDA Collaboration), Improved Limit on Neutrinoless Double- $\beta$ Decay of ${ }^{76} \mathrm{Ge}$ from GERDA Phase II, Phys. Rev. Lett. 120, 132503 (2018).

[4] C. E. Aalseth et al. (Majorana Collaboration), Search for Neutrinoless Double- $\beta$ Decay in ${ }^{76} \mathrm{Ge}$ with the Majorana Demonstrator, Phys. Rev. Lett. 120, 132502 (2018).

[5] C. Alduino et al. (CUORE Collaboration), First Results from CUORE: A Search for Lepton Number Violation Via $0 \nu \beta \beta$ Decay of ${ }^{130}$ Te, Phys. Rev. Lett. 120, 132501 (2018).

[6] J. B. Albert et al. (EXO Collaboration), Search for Neutrinoless Double-Beta Decay with the Upgraded EXO-200 Detector, Phys. Rev. Lett. 120, 072701 (2018).

[7] R. Arnold et al. (NEMO-3 Collaboration), Measurement of the $2 \nu \beta \beta$ decay half-life and search for the $0 \nu \beta \beta$ decay of ${ }^{116} \mathrm{Cd}$ with the NEMO-3 detector, Phys. Rev. D 95, 012007 (2017).

[8] H. K. Dreiner, H. E. Haber, and S. P. Martin, Twocomponent spinor techniques and Feynman rules for quantum field theory and supersymmetry, Phys. Rep. 494, 1 (2010).

[9] P. F. de Salas, D. V. Forero, C. A. Ternes, M. Tortola, and J. W. F. Valle, Status of neutrino oscillations 2018: $3 \sigma$ hint for normal mass ordering and improved CP sensitivity, Phys. Lett. B 782, 633 (2018).

[10] P.-H. Gu and H.-J. He, Neutrino mass and baryon asymmetry from Dirac seesaw, J. Cosmol. Astropart. Phys. 12 (2006) 010.

[11] C.-Y. Yao and G.-J. Ding, Systematic analysis of Dirac neutrino masses from a dimension five operator, Phys. Rev. D 97, 095042 (2018).

[12] M. Roncadelli and D. Wyler, Naturally Light Dirac Neutrinos in Gauge Theories, Phys. Lett. 133B, 325 (1983).

[13] P. Roy and O. U. Shanker, Observable Neutrino Dirac Mass and Supergrand Unification, Phys. Rev. Lett. 52, 713 (1984); Erratum, Phys. Rev. Lett.52, 2190(E) (1984).

[14] P.-H. Gu, H.-J. He, and U. Sarkar, Realistic neutrinogenesis with radiative vertex correction, Phys. Lett. B 659, 634 (2008).
[15] E. Ma and R. Srivastava, Dirac or inverse seesaw neutrino masses with $B-L$ gauge symmetry and $S_{3}$ flavor symmetry, Phys. Lett. B 741, 217 (2015).

[16] E. Ma and O. Popov, Pathways to naturally small Dirac neutrino masses, Phys. Lett. B 764, 142 (2017).

[17] S. C. Chuliá, R. Srivastava, and J. W. F. Valle, Seesaw roadmap to neutrino mass and dark matter, Phys. Lett. B 781, 122 (2018).

[18] S. C. Chuliá, R. Srivastava, and J. W. F. Valle, Seesaw Dirac neutrino mass through dimension-six operators, Phys. Rev. D 98, 035009 (2018).

[19] N. Bernal, D. Restrepo, C. Yaguna, and Ó. Zapata, Twocomponent dark matter and a massless neutrino in a new $B-L$ model, Phys. Rev. D 99, 015038 (2019).

[20] W. Wang, R. Wang, Z.-L. Han, and J.-Z. Han, The $B-L$ scotogenic models for Dirac neutrino masses, Eur. Phys. J. C 77, 889 (2017).

[21] Z.-L. Han and W. Wang, $Z^{\prime}$ portal dark matter in $B-L$ scotogenic Dirac model, Eur. Phys. J. C 78, 839 (2018).

[22] P. Batra, B. A. Dobrescu, and D. Spivak, Anomaly-free sets of fermions, J. Math. Phys. (N.Y.) 47, 082301 (2006).

[23] D. Nanda and D. Borah, Common origin of neutrino mass and dark matter from anomaly cancellation requirements of a $U(1)_{B-L}$ model, Phys. Rev. D 96, 115014 (2017).

[24] V. Silveira and A. Zee, Scalar phantoms, Phys. Lett. 161B, 136 (1985).

[25] J. McDonald, Gauge singlet scalars as cold dark matter, Phys. Rev. D 50, 3637 (1994).

[26] C. P. Burgess, M. Pospelov, and T. ter Veldhuis, The minimal model of nonbaryonic dark matter: A singlet scalar, Nucl. Phys. B619, 709 (2001).

[27] N. G. Deshpande and E. Ma, Pattern of symmetry breaking with two Higgs doublets, Phys. Rev. D 18, 2574 (1978).

[28] R. Barbieri, L. J. Hall, and V. S. Rychkov, Improved naturalness with a heavy Higgs: An alternative road to LHC physics, Phys. Rev. D 74, 015007 (2006).

[29] M. Kadastik, K. Kannike, and M. Raidal, Matter parity as the origin of scalar Dark Matter, Phys. Rev. D 81, 015002 (2010).

[30] M. Kadastik, K. Kannike, and M. Raidal, Dark matter as the signal of grand unification, Phys. Rev. D 80, 085020 (2009); Erratum, Phys. Rev. D 81, 029903(E) (2010).

[31] M. Kakizaki, A. Santa, and O. Seto, Phenomenological signatures of mixed complex scalar WIMP dark matter, Int. J. Mod. Phys. A 32, 1750038 (2017). 
[32] X. Liu and L. Bian, Dark matter and electroweak phase transition in the mixed scalar dark matter model, Phys. Rev. D 97, 055028 (2018).

[33] Y. G. Kim and K. Y. Lee, The minimal model of fermionic dark matter, Phys. Rev. D 75, 115012 (2007).

[34] C. E. Yaguna, Singlet-doublet Dirac dark matter, Phys. Rev. D 92, 115002 (2015).

[35] M. Carena, A. Daleo, B. A. Dobrescu, and T. M. P. Tait, $Z^{\prime}$ gauge bosons at the Tevatron, Phys. Rev. D 70, 093009 (2004).

[36] G. Cacciapaglia, C. Csáki, G. Marandella, and A. Strumia, The minimal set of electroweak precision parameters, Phys. Rev. D 74, 033011 (2006).

[37] M. Aaboud et al. (ATLAS Collaboration), Search for new high-mass phenomena in the dilepton final state using $36 \mathrm{fb}^{-1}$ of proton-proton collision data at $\sqrt{s}=13 \mathrm{TeV}$ with the ATLAS detector, J. High Energy Phys. 10 (2017) 182.

[38] M. Escudero, S. J. Witte, and N. Rius, The dispirited case of gauged $\mathrm{U}(1)_{B-L}$ dark matter, J. High Energy Phys. 08 (2018) 190.

[39] A. M Sirunyan et al. (CMS Collaboration), Search for highmass resonances in dilepton final states in proton-proton collisions at $\sqrt{s}=13 \mathrm{TeV}$, J. High Energy Phys. 06 (2018) 120.

[40] T. Nomura and H. Okada, Neutrinophilic two Higgs doublet model with dark matter under an alternative $U(1)_{B-L}$ gauge symmetry, Eur. Phys. J. C 78, 189 (2018).
[41] S. Kanemura, T. Nabeshima, and H. Sugiyama, Neutrino Masses from Loop-Induced Dirac Yukawa Couplings, Phys. Lett. B 703, 66 (2011).

[42] P. Fileviez Perez and C. Murgui, Sterile neutrinos and B-L symmetry, Phys. Lett. B 777, 381 (2018).

[43] S. Weinberg, Goldstone Bosons as Fractional Cosmic Neutrinos, Phys. Rev. Lett. 110, 241301 (2013).

[44] F. Staub, SARAH 4: A tool for (not only SUSY) model builders, Comput. Phys. Commun. 185, 1773 (2014).

[45] P. O. Ludl and W. Grimus, A complete survey of texture zeros in the lepton mass matrices, J. High Energy Phys. 07 (2014) 090; Erratum, J. High Energy Phys. 10 (2014) 126(E).

[46] A. M. Baldini et al. (MEG Collaboration), Search for the lepton flavour violating decay $\mu^{+} \rightarrow \mathrm{e}^{+} \gamma$ with the full dataset of the MEG experiment, Eur. Phys. J. C 76, 434 (2016).

[47] P.-H. Gu and U. Sarkar, Radiative neutrino mass, dark matter and leptogenesis, Phys. Rev. D 77, 105031 (2008).

[48] Y. Farzan and E. Ma, Dirac neutrino mass generation from dark matter, Phys. Rev. D 86, 033007 (2012).

[49] M. Reig, D. Restrepo, J. W. F. Valle, and O. Zapata, Boundstate dark matter and Dirac neutrino masses, Phys. Rev. D 97, 115032 (2018).

[50] V. De Luca, A. Mitridate, M. Redi, J. Smirnov, and A. Strumia, Colored dark matter, Phys. Rev. D 97, 115024 (2018).

[51] C. Bonilla, S. C. Chuliá, R. Cepedello, E. Peinado, and R. Srivastava, Dark matter stability and Dirac neutrinos using only Standard Model symmetries, arXiv:1812.01599. 